\title{
THE EQUILONG TRANSFORMATIONS OF EUCLIDEAN SPACE*
}

BY

\author{
B. H. BROWN
}

1.

In the classical non-euclidean geometries of space of $\boldsymbol{n}$ dimensions, distance as well as angle has a projective definition, and equilong transformations are the dual of conformal transformations by polar reciprocation in the absolute. In euclidean space the projective definition is lost, but while the preceding duality breaks down, Schefferst exhibited a perfect analogy in the euclidean plane by the use of the dual numbers of Study. We know that for any function of the complex variable

$$
f(x+i y)=X(x, y)+i Y(x, y), \quad i^{2}=-1
$$

where $X$ and $Y$ satisfy the Cauchy-Riemann differential equations

$$
\frac{\partial X}{\partial x}=\frac{\partial Y}{\partial y}, \quad \frac{\partial X}{\partial y}=-\frac{\partial Y}{\partial x}
$$

the point transformation

$$
X=X(x, y), \quad Y=Y(x, y)
$$

is directly conformal. Scheffers proved that if $u$ and $v$ denote the Hessian normal coordinates of an oriented line ( $v$ the distance parameter), for any function of the dual number

$$
f(u+\varepsilon v)=U(u, v)+\varepsilon V(u, v), \quad \varepsilon^{2}=0,
$$

where $U$ and $V$ satisfy the differential equations

$$
\frac{\partial U}{\partial u}=\frac{\partial V}{\partial v}, \quad \frac{\partial U}{\partial v}=0
$$

* Presented to the Society, December 27, 1922.

† Mathematische Annalen, vol. 60 (1905), p. 491. 
the oriented line transformation

$$
U=U(u, v), \quad \nabla=\nabla(u, v)
$$

is directly equilong.

Since the conformal group in non-euclidean as well as in euclidean threespace is a ten-parameter group, the equilong group in non-euclidean threespace depends on ten parameters. But the equilong group in euclidean space contains arbitrary functions.* In space of more than three dimensions, the conformal euclidean group, and the conformal and equilong non-euclidean groups contain a finite number of parameters, but Coolidget has shown that: The most general equilong transformation of a euclidean space of $n$ dimensions depends on the most general conformal transformation of a space of $n-1$ dimensions and an arbitrary function of the direction parameters. The distance parameter enters linearly.

The above theorem is true for $n \geqq 3$, but the last statement is also true for $n=2$, since the integration of (3) gives

$$
U=U(u), \quad V=U^{\prime} \cdot v+U_{1}(u)
$$

This fact leads to a hitherto unnoticed analogy between the conformal and equilong transformations in the plane, and to a sharpening of the contrast in higher spaces. The functions $X$ and $Y$ of (1) satisfy Laplace's equation. Again in Study's formulation of the conformal (and therefore equilong) transformations in the Riemannian and Lobatschewskian planes; the functions of hypercomplex variables are separable into functions satisfying either Laplace's equation or the hyperbolic form

$$
\frac{\partial^{2} \Phi}{\partial x^{2}}-\frac{\partial^{2} \Phi}{\partial y^{2}}=0
$$

\footnotetext{
* This remarkable theorem was first enunciated, without proof, by Study, Sitzungs berichte der Niederrheinischen Gesellschaft für Natur-und Heilkunde, Dec. 5, 1904. In 1908 Coolidge gave the first published proof of this theorem, and a correct explicit form for these transformations in these Transactions, vol. 9 (1908), p. 178. An incorrect derivation leading to a ten-parameter group was given by Loehrl in his Würzburg dissertation (1910). A demonstration, independent of Coolidge's, was given by Blaschke, Archiv der Mathematik und Physik, vol. 16 (1910), p. 182. The final form of these transformations is, however, incorrect with respect to a distinction of signs. This error has never, to our knowledge, been corrected. In 1916 Coolidge in his Treatise on the Circle and the Sphere, p. 419, changing the correct form of his 1908 paper, reproduced Blaschke's incorrect form.

+ Loc. cit., p. 182.
} 
equations which are not essentially distinct for complex solutions. Finally the functions $U$ and $V$ of (4) satisfy the parabolic equation

$$
\frac{\partial^{2} \Phi}{\partial v^{2}}=0
$$

But while the functions in the equations of an equilong transformation in $n$-dimensional euclidean space are non-trivial solutions of (5) $(v$ denoting the distance parameter), the analogy is completely lost in the other cases.

In this paper we give in Section 2 a new demonstration of the fundamental equations for equilong transformations in euclidean three-space. The main portion of this paper is then devoted to a discussion of groups of these transformations which leave invariant various differential expressions and equations.

The equation

2.

$$
\frac{u+v}{1+u v} x-\frac{i(u-v)}{1+u v} y+\frac{1-u v}{1+u v} z=\frac{u}{1+u v}
$$

represents an oriented plane such that the direction cosines of its oriented normal are the coefficients of $x, y$, and $z$, and such that the distance from the origin to the plane is $\frac{w}{1+u v}$. Then $u, v, w$ are Bonnet* tangential coordinates of this oriented plane. Exceptional cases occur when: $(a) 1+u v=0$ (minimal plane); (b) the spherical representation of the plane is a point on a ruling through the south pole. The point of contact of a plane and any envelope which touches the plane is given by the equations

$$
\begin{aligned}
(u+v) x-i(u-v) y+(1-u v) z & =w, \\
x-i y-v z & =\frac{\partial w}{\partial u}=p, \\
x+i y-u z & =\frac{\partial w}{\partial v}=q .
\end{aligned}
$$

The square of the distance between two points of a plane is

$$
\left(p_{1}-p_{2}\right)\left(q_{1}-q_{2}\right)
$$

*Liouville's Journal, ser. 2, vol. 5 (1860), pp. 153-266. 
In any plane transformation

(9)

$$
\begin{aligned}
U & =U(u, v, w), \\
\nabla & =\nabla(u, v, w), \\
W & =W(u, v, w), \quad J \neq 0,
\end{aligned}
$$

corresponding points of two corresponding planes are projectively related. To find the equations of the equilong transformations we simplify the form of (9) by imposing the necessary conditions that the collineation be

(a) affine;

(b) directly or indirectly conformal.

Blaschke has shown that under these impositions the once-extended transformations are

$$
\begin{aligned}
& \text { Direct: } U=U(u) \text {, } \\
& \text { Indirect: } U=U(v) \text {, } \\
& \nabla=V(v), \\
& V=V(u) \\
& W=W(u, v, w), \\
& W=W(u, v, w) \\
& P=\frac{1}{U^{\prime}}\left(\frac{\partial W}{\partial w} p+\frac{\partial W}{\partial u}\right) \\
& P=\frac{1}{\nabla^{\prime}}\left(\frac{\partial W}{\partial w} q+\frac{\partial W}{\partial v}\right) \\
& Q=\frac{1}{\nabla^{\prime}}\left(\frac{\partial W}{\partial w} q+\frac{\partial W}{\partial v}\right) \\
& Q=\frac{1}{U^{\prime}}\left(\frac{\partial W}{\partial w} p+\frac{\partial W}{\partial u}\right)
\end{aligned}
$$

It is now necessary and sufficient to impose the condition that

$$
\left(p_{1}-p_{2}\right)\left(q_{1}-q_{2}\right)
$$

be an absolute invariant. In either case we have

$$
\left(P_{1}-P_{2}\right)\left(Q_{1}-Q_{2}\right)=\frac{1}{U^{\prime} \nabla^{\prime}}\left(\frac{\partial W}{\partial w}\right)^{2}\left(p_{1}-p_{2}\right)\left(q_{1}-q_{2}\right)=\left(p_{1}-p_{2}\right)\left(q_{1}-q_{2}\right)
$$

Hence

$$
\frac{\partial W}{\partial w}=\sqrt{U^{\prime} V^{\prime}}
$$


We thus have as our fundamental equations

Direct: $U=U(u), \quad$ Indirect: $U=U(v)$,

$$
\begin{array}{ll}
V=V(v), & V=V(u), \\
W=\sqrt{U^{\prime} \nabla^{\prime}} w+F(u, v) ; & W=V \overline{U^{\prime} \nabla^{\prime}} w+F(u, v) .
\end{array}
$$

Blaschke, and subsequent writers, incorrectly insert a \pm sign under the radicals of (10). For such transformations the plane projectivity is either directly conformal and indirectly equiareal, or indirectly conformal and directly equiareal. In neither of these cases is square of distance preserved.

3.

We state, without proof, the fundamental formulas in the differential geometry of a non-developable oriented surface $w=w(u, v)$. The corrdinates of a point of tangency are given by

$$
\begin{aligned}
x+i y & =\frac{u w-p u^{2}+q}{1+u v}, \\
x-i y & =\frac{v w-q v^{2}+p}{1+u v}, \\
z & =\frac{w-u p-v q}{1+u v} .
\end{aligned}
$$

Let $\frac{\partial^{2} w}{\partial u^{2}}=r, \frac{\partial^{2} w}{\partial u \partial v}=s, \frac{\partial^{2} w}{\partial v^{2}}=t ;$ then the three fundamental forms are

$$
\begin{aligned}
d s^{2}= & r(z+s) d u^{2}+\left\{(z+s)^{2}+r t\right\} d u d v+t(z+s) d v^{2} \\
& -\frac{r}{1+u v} d u^{2}-\frac{2(z+s)}{1+u v} d u d v-\frac{t}{1+u v} d v^{2} \\
d \sigma^{2}= & \frac{4 d u d v}{(1+u v)^{2}}
\end{aligned}
$$

The total curvature is

$$
\frac{-4}{(1+u v)^{2}\left\{r t-(z+s)^{2}\right\}}
$$


the mean curvature

$$
\frac{4(z+s)}{(1+u v)\left\{r t-(z+s)^{2}\right\}}
$$

the lines of curvature are given by

$$
r d u^{2}-t d v^{2}=0
$$

the radii of principal curvature by

$$
R=\frac{-w+u p+v q-(1+u v)(s+\sqrt{r t})}{2}
$$

and the centers of curvature by

$$
\begin{aligned}
X+i Y & =q-u(s+\sqrt{r t}) \\
X-i Y & =p-v(s+\sqrt{r t}) \\
Z & =\frac{w-u p-v q-(1-u v)(s+\sqrt{r t})}{2} .
\end{aligned}
$$

The differential equations of minimal curves and of asymptotic curves follow from (12). The differential equation of minimal surfaces is

$$
z+s=0, \quad r t \neq 0
$$

of spheres (oriented, non-null spheres)

$$
r=t=0, \quad z+s \neq 0
$$

of points, which are not to be excluded on the score of the discriminant of the first quadratic form vanishing, but which are proper envelopes of $\infty^{2}$ planes, and may be regarded either as minimal surfaces or as spheres,

$$
r=t=z+s=0
$$

4.

Among the indirect equilong transformations, the analogue of the identity is that one which merely reverses the orientation of a plane, without changing 
its position. This transformation we term the "pseudo-identity". It is clear that every indirect transformation is the product of a direct transformation and the pseudo-identity. The twice-extended form of the pseudo-identity is

$$
\begin{aligned}
& U=-\frac{1}{v}, \\
& V=-\frac{1}{u}, \\
& W=-\frac{w}{u v}, \\
& P=\frac{w-v q}{u}, \\
& Q=\frac{w-u p}{v}, \\
& R=-\frac{v^{s} t}{u}, \\
& S=u p+v q-w-u v s, \\
& T=-\frac{u^{s} r}{v} .
\end{aligned}
$$

It will be observed that the equations and expressions (11) to (20) are invariant (invariant except for sign) under (21) as they have geometric significance independent of (dependent on) orientation. The differential geometry of oriented surfaces is the interpretation of the differential invariants of the extended pseudo-identity. A surface whose equation is invariant under the pseudo-identity is obviously one-sided. We have then the

THEOREM. A necessary and sufficient condition that a surface $w=f(u, v)$ be one-sided is that $f$ satisfy the functional equation $f(u, v)=-u v f\left(-\frac{1}{v},-\frac{1}{u}\right)$.

We shall next prove the

5.

THEOREM. Any oriented non-developable surface may, by each of two and only two distinct, direct equilong transformations, be transformed into any other oriented non-developable surface, and that with an arbitrary analytic directly conformal mapping of their spherical representations.

This theorem was suggested by Study in his 1904 paper, but he stated, incorrectly, that there was one and only one such transformation. 
Let us consider two surfaces

$$
\begin{gathered}
w=f_{1}(U, V), \\
w=f_{2}(u, v),
\end{gathered}
$$

and let us assume that the spherical representation of the first $(U, V)$ is conformally mapped on the spherical representation of the second $(u, v)$ by the directly conformal transformation

$$
\begin{gathered}
U=U(u), \\
V=V(v) .
\end{gathered}
$$

The theorem is proved if, in the group of transformations.

$$
\begin{aligned}
& U=U(u), \\
& V=V(v), \\
& W=\sqrt{U^{\prime} V^{\prime}} w+F(u, v),
\end{aligned}
$$

we can determine two and only two functions $F(u, v)$ such that the first surface is transformed into the second. This means that

$$
\sqrt{\bar{U}^{\prime} \nabla^{\prime}} w+F(u, v)=f_{1}(U(u), V(v))
$$

must be identical with

$$
w=f_{\mathbf{2}}(u, v)
$$

which is true when and only when

$$
F(u, v)=f_{1}(U(u), V(v))-\sqrt{U^{\prime} \nabla^{\prime}} f_{2}(u, v)
$$

hence there are always two distinct transformations. We should note that there is no exception when $f_{2}=0$.

6.

The twice-extended form of the general direct equilong transformation may be written 


$$
\begin{aligned}
& U=U(u), \\
& \nabla=V(v), \\
& W=V \overline{U^{\prime} V^{\prime}} w+F(U, \nabla), \\
& P=\frac{w V^{\prime \frac{1}{2}} U^{\prime \prime}}{2 U^{\frac{8}{2}}}+\frac{p V^{\prime \frac{1}{2}}}{U^{\prime \frac{1}{2}}}+F_{U},
\end{aligned}
$$

(22)

$$
\begin{aligned}
& Q=\frac{w U^{\frac{1}{2}} V^{\prime \prime}}{2 V^{\prime \frac{8}{2}}}+\frac{q U^{\frac{1}{2}}}{{V^{\prime}}^{\frac{1}{2}}}+F_{V}, \\
& R=\frac{w V^{\frac{1}{2}} U^{\prime \prime \prime}}{2 U^{\prime \frac{5}{2}}}-\frac{3}{4} \frac{w U^{\prime 2} V^{\prime \frac{1}{2}}}{U^{\prime \frac{7}{2}}}+\frac{r V^{\prime \frac{1}{2}}}{U^{\frac{8}{2}}}+F_{U U}, \\
& S=\frac{w}{4\left(U^{\prime \prime} V^{\prime \prime}\right.}+\frac{\left.p V^{\prime \prime}\right)^{\frac{8}{2}}}{2 U^{\prime \frac{1}{2}} V^{\prime \frac{8}{2}}}+\frac{q U^{\prime \prime}}{2 V^{\frac{1}{2}} U^{\frac{3}{2}}}+\frac{s}{\left(U^{\prime} V^{\prime}\right)^{\frac{1}{2}}}+F_{V V} \\
& T=\frac{w U^{\prime \frac{1}{2}} V^{\prime \prime \prime}}{2 V^{\frac{5}{2}}}-\frac{3}{4} \frac{w V^{\prime 2} U^{\prime \frac{1}{2}}}{\nabla^{\prime \frac{7}{2}}}+\frac{t U^{\prime \frac{1}{2}}}{V^{\prime \frac{3}{2}}}+F_{V V} .
\end{aligned}
$$

It is proposed to discuss the invariance of the equations and expressions (11) to (20) under (22).

First, under a direct transformation, a necessary and sufficient condition that spheres transform into spheres is that $R$ vanish with $r$ and $T$ with $t$.

This rèquires

$$
\begin{aligned}
2 U^{\prime} U^{\prime \prime \prime}-3 U^{\prime 2} & =0, \\
2 V^{\prime} V^{\prime \prime \prime}-3 V^{\prime \prime 2} & =0, \\
F_{U U}=F_{V V} & =0 .
\end{aligned}
$$

The first two of (23) recall the Schwarzian derivative. Integrating, we have

$$
\begin{aligned}
& U=\frac{\alpha u+\beta}{\gamma u+\delta}, \quad V=\frac{\alpha^{\prime} v+\beta^{\prime}}{\gamma^{\prime} v+\delta^{\prime}}, \\
& F=A U V+B U+C V+D .
\end{aligned}
$$


We may and shall choose ratios so that

$$
\alpha \boldsymbol{\delta}-\beta \gamma=\alpha^{\prime} \delta^{\prime}-\beta^{\prime} \gamma^{\prime}=1
$$

We have then

$$
W=\frac{ \pm w+a u v+b u+c v+d}{(\gamma u+\delta)\left(\gamma^{\prime} v+\delta^{\prime}\right)}
$$

(24) and (25) giving the equations of the well known Laguerre group. We might just as easily have found these by imposing the condition that lines (more properly strips) of curvature go into lines of curvature.

It is easy to verify that translations are given by

$$
U=u, \quad V=v, \quad W=w+a(u v-1)+b u+c v
$$

reflection in the origin by

$$
U=u, \quad V=v, \quad W=-w
$$

dilatations by

$$
U=u, \quad \nabla=v, \quad W=w+a(u v+1)
$$

rotations by

$$
U=\frac{a u+b}{c u+d}, \quad V=\frac{d v-c}{-b v+a}, \quad W=\frac{w}{(c u+d)(-b v+a)},
$$

where $a d-b c=1$. Other transformations involve Laguerre inversions.

On account of the simplicity and frequency of occurrence of the expression $z+s$, we next consider the transformations under which minimal surfaces transform into minimal surfaces. It is clear that any transformation

$$
U=u, \quad \nabla=v, \quad W=w+f_{1}(u, v),
$$

where $f_{1}$ is a solution of $z+s=0$, will carry any minimal surface into a minimal surface, for the sum of two solutions of a linear homogeneous partial differential equation is itself a solution. To this group of transformations we may, from geometric considerations, adjoin (27) and (29). It turns out that these are the only such transformations; we term this group the "minimal group". 
To prove this statement, if we impose $Z+S=0$ on $z+s=0$ we must have

$$
\begin{gathered}
w\left[{U^{\prime 2}}^{\prime 2}-\frac{1}{2} U \nabla^{\prime 2} U^{\prime \prime}-\frac{1}{2} \nabla U^{\prime 2} V^{\prime \prime}+\frac{1}{4} U^{\prime \prime} \nabla^{\prime \prime}+\frac{1}{4} U^{\prime \prime} V^{\prime \prime} U^{\prime} \nabla^{\prime}\right] \\
+p\left[-U U^{\prime} \nabla^{\prime 2}+\frac{1}{2} U^{\prime} \nabla^{\prime \prime}+\frac{1}{2} U U^{\prime} \nabla \nabla^{\prime \prime}\right]
\end{gathered}
$$

(30)

$$
\begin{aligned}
& +q\left[-\nabla V^{\prime} U^{\prime 2}+\frac{1}{2} \nabla^{\prime} U^{\prime \prime}+\frac{1}{2} \nabla \nabla^{\prime} U U^{\prime \prime}\right] \\
& +s\left[U^{\prime} V^{\prime}+U \nabla U^{\prime} \nabla^{\prime}\right] \\
& +\left(U^{\prime} \nabla^{\prime}\right)^{\frac{8}{2}}\left[F-U F_{U}-\nabla F_{\nabla}+(1+U \nabla) F_{\sigma \nabla}\right] \\
& \equiv \frac{U^{\prime} \nabla^{\prime}(1+U \nabla)}{1+u v}\{w-u p-v q+(1+u v) s\} .
\end{aligned}
$$

Hence it is necessary that

$$
\begin{aligned}
& -\frac{U V^{\prime}}{1+U V}+\frac{1}{2} \frac{V^{\prime \prime}}{\nabla^{\prime}}=-\frac{u}{1+u v} \\
& -\frac{\nabla U^{\prime}}{1+U V}+\frac{1}{2} \frac{U^{\prime \prime}}{U^{\prime}}=-\frac{v}{1+u v}
\end{aligned}
$$

Subtracting (33) from the product of (31) and (32) we have

$$
\frac{U^{\prime} \nabla^{\prime}}{(1+U V)^{2}}=\frac{1}{(1+u v)^{2}}
$$


hence we may rewrite (31) and (32) as

$$
u=\frac{U V^{\frac{1}{2}}}{U^{\frac{1}{2}}}-\frac{1}{2}(1+U V) \frac{V^{\prime \prime}}{V^{\frac{8}{2}} U^{\frac{1}{2}}},
$$

$$
v=\frac{V U^{\prime \frac{1}{2}}}{V^{\frac{1}{2}}}-\frac{1}{2}(1+U V) \frac{U^{\prime \prime}}{U^{\frac{8}{2}} V^{\prime \frac{1}{2}}} .
$$

Differentiating the first of (36) with regard to $v$, and the second with regard to $u$,

$$
\begin{aligned}
& \frac{U(1+U V)}{V^{\prime \frac{5}{2}}}\left\{V^{\prime \prime \prime} \nabla^{\prime}-\frac{3}{2} V^{\prime 2}\right\}=0 \\
& \frac{V(1+U V)}{U^{\prime \frac{5}{2}}}\left\{U^{\prime \prime \prime} U^{\prime}-\frac{3}{2} U^{\prime \prime 2}\right\}=0
\end{aligned}
$$

hence

$$
U=\frac{\alpha u+\beta}{\gamma u+\delta}, \quad V=\frac{\alpha^{\prime} v+\beta^{\prime}}{\gamma^{\prime} v+\delta^{\prime}}
$$

are necessary conditions on $U$ and $V$. Now if, in (33), we substitute the value of $(1+u v)$ given in (35), and these last values of $U$ and $V$, it is necessary that

$$
\alpha: \beta: \gamma: \delta=\delta^{\prime}:-\gamma^{\prime}:-\beta^{\prime}: \alpha^{\prime},
$$

and the theorem is proved.

If we examine the form of any one of (12), (13), or (14) we see without difficulty that any one of them is invariant if and only if the transformation belongs to both the Laguerre and minimal groups. The actual verification of this is so much a repetition of the previous proof that it is omitted. Unfortunately the only such transformations are the congruent transformations; for the only non-parallel transformations of the minimal group are rotations, and of the parallel transformations of the Laguerre group, dilatations carry a point into a sphere which is not a minimal surface. Our results are then essentially negative if we impose the condition on all surfaces, but there are interesting special cases for groups of transformations and groups of surfaces. 
We consider only one such example: the conformal mapping of surfaces under equilong transformations. The differential equation of minimal curves on a surface is, by (12),

$$
r(z+s) d u^{2}+\left\{(z+s)^{2}+r t\right\} d u d v+t(z+s) d v^{2}=0 .
$$

The following theorems follow immediately:

1. Under a transformation of the Laguerre group any sphere and the transformed sphere are conformally mapped.

2. Under a transformation of the minimal group, any minimal surface and its transformed minimal surface are conformally mapped.

3. The only surfaces transformed into their spherical representations with conformal mapping by equilong transformations are spheres and minimal surfaces.

4. A minimal surface may be transformed into any sphere with conformal mapping, and conversely.

7.

Since the solutions of linear homogeneous partial differential equations possess the additive property, we may associate with every such equation the surfaces that are solutions thereof, and a corresponding group of direct parallel equilong transformations

$$
U=u, \quad V=v, \quad W= \pm w+f(u, v)
$$

where $f$ is itself a solution of the given differential equation; under this group of transformations the surfaces are permuted among themselves. Obviously we shall be most interested in differential equations invariant under the pseudo-identity. For such differential equations there is a sub-group of transformations which will permute the one-sided surfaces of the group among themselves, for the solutions of the linear homogeneous functional equation

$$
f(u, v)=-u v f\left(-\frac{1}{v},-\frac{1}{u}\right)
$$

possess the additive property. Thus, for example, the double minimal surfaces are permuted among themselves by the appropriate subgroup, for they are the only one-sided minimal surfaces.

Let us consider a solution of (18), a minimal surface whose equation may be written

$$
w=2 v f(u)+2 u f_{1}(v)-(1+u v)\left[f^{\prime}(u)+f_{1}^{\prime}(v)\right] .
$$


We may associate with this a two-parameter family of minimal surfaces $[A, B]$

$$
w=2 v A f+2 u B f_{1}-(1+u v)\left[A f^{\prime}+B f_{1}^{\prime}\right],
$$

where $[A, A]$ are expansions of the original surface, and $[A, 1 / A]$ its continuous deforms; ${ }^{*}$ and we may also associate therewith the two-parameter family of parallel equilong transformations of the minimal group which permute these among themselves.

The $\infty^{2}$ points, one from each of these surfaces, with properly parallel tangent planes (planes with the same $u$ and $v$ ) may be obtained by expanding at the origin the conic which is the path-curve of the point of the original minimal surface under the continuous transformation which gives the associated surfaces. These points are coplanar, as the plane of the conic contains the origin. The tangent planes are not, in general, coincident with this locus plane. Under a parallel equilong transformation any aggregate of $\infty^{8}$ planar elements with (properly) parallel planes are rigidly translated as a whole (Study and Blaschke) so that an equilong transformation of the group effects a translation of this plane. In this plane associated minimal surfaces are represented by points of a conic which is one of a one-parameter family of homothetic conics. A transformation of the group will translate this conic, the transformed conic cutting the original conic and each of the $\infty^{1}$ homothetic conics in two points (since the axes of the conics are parallel). Hence under any transformation of the group only two associated surfaces of a given minimal surface will transform into associated surfaces; the other associated surfaces will, in general, be transformed by pairs into associated minimal surfaces of the $\infty^{1}$ expansions of the given minimal surface.

This discussion may obviously be extended to any system of surfaces whose equations are

$$
w=A \sum f_{i}(u, v) F^{[i]}(u)+B \sum g_{i}(u, v) G^{[i]}(v),
$$

except that the surfaces $[A, 1 / A]$ are not generally continuous deforms.

As an example we have certain surfaces of Goursat, $f$ for which the sum of the radii of curvature at a point is proportional to the distance from the

* Although not coextensive, we shall use the expression "continuous deforms" as equivalent to "associrted surfaces". This is proper, since a continuous deform is an associated surface, or can be made to coincide with one by a congruent transformation.

† American Journal of Mathematics, vol. 10 (1887-8), p. 187; Baroni, Giornale di Matematiche, vol. 28 (1890), p. 349. 
origin to the tangent plane at the point. From (6) and (16) the equation of these surfaces is

$$
(1+u v) s-u p-v q+w\left[1+\frac{2 k}{1+u v}\right]=0
$$

the ratio of proportionality being $2 k$. Goursat proved that when (and only when) $m$ defined by

$$
k=\frac{(m+1)(m-2)}{2}
$$

is integral, the solution of (39) may be obtained free from quadratures, and, indeed, in the form (38). For these values of $k$ the preceding discussion holds, with deletion of the expression "continuous deforms".

Two special cases are worthy of note:

(a) If $k=0$, (39) is the differential equation of minimal surfaces;

(b) If $k=-1,(39)$ is the differential equation of Appell* surfaces for which the projection of the origin on every normal is midway between the centers of principal curvature.

In the papers of Appell and Goursat, we find three classical transformations:

(a) A transformation of Appell which carries a particular minimal surface into an Appell surface.

(b) A transformation of Appell which carries a particular Bonnett surface into an Appell surface;

(c) A transformation of Goursat which carries a particular Goursat surface into another Goursat surface with change of $k$. These transformations are equilong, and, in fact, special cases of Study's theorem where the mapping of the spherical representations is the identity, and the upper sign for the radical is used.

The general methods of this section are applicable to a large class of surfaces defined by some relation involving their radii of curvature. One further group of transformations, defined by a linear non-homogeneous partial differential equation, merits attention. From (16) it follows immediately that

$$
(1+u v) s-u p-v q+w=-2 k
$$

*American Journal of Mathematics, vol. 10 (1887-8), p. 175.

† Paris Comptes Rendus, vol. 42 (1856), p. 119, Note sur les surfaces pour lesquelles la somme des deux rayons de courbure principaux est égale au double de la normale. 
is the differential equation of all surfaces for which the sum of the radii of principal curvature is a constant $2 k$. Such a surface is, for example, the "inner" surface of a sphere of radius $k$, center at the origin

$$
w=-k(1+u v) \text {. }
$$

Thus knowing one particular solution of (40) we obtain all the other solutions by adding to the right-hand side of (41) the general solution of the differential equation for minimal surfaces. Hence the direct parallel equilong transformations

$$
U=u, \quad V=v, \quad W=w+f(u, v),
$$

where $f(u, v)$ satisfies (40), carry minimal surfaces into the surfaces we are considering, and carry surfaces the sum of whose radii of principal curvature is $2 k_{1}$ into surfaces the sum of whose radii of principal curvature is $2\left(k+k_{1}\right)$.

DarTMOUTH COLLEGE,

HANOVER, N. H. 